\title{
Estrategias preventivas del abuso de alcohol
}

\author{
Calafat, A.
}

Socidrogalcohol. Irefrea.

Enviar correspondencia: Amador Calafat. Rambla 15, 2 $3^{\mathrm{a}}$. 07003 Palma de Mallorca. adicciones@socidrogalcohol.org

\section{RESUMEN}

La prevención de los problemas relacionados con el consumo de alcohol sigue siendo una gran prioridad de la salud pública, a pesar de un cierto descenso en el consumo global, en especial porque la embriaguez sigue siendo una forma de consumo muy frecuente los fines de semana. Existe en la actualidad suficiente investigación para avalar las diversas medidas que se han venido aplicando, estando entre las más eficaces disminuir el fácil acceso a su consumo. Pero hay otras medidas que se han mostrado útiles como disminuir la asociación entre conducir y beber, controlar la publicidad,.. Hay medidas clásicas como la prevención escolar que necesitan de más investigación sobre todo dirigida a avalar los resultados. Y hay nuevas actuaciones dirigidas especialmente a disminuir los efectos de los problemas derivados del consumo de alcohol, a buscar alternativas como salir a divertirse sin consumir alcohol, o a intervenir sobre los contextos recreativos, tanto desde un punto de vista cultural como del contexto físico que pueden ser prometedoras, pero que también requerirán que se evalúen convenientemente.

Palabras clave: prevención, alcohol, impuestos, precios, evaluación, revisión.

\section{SUMMARY}

The prevention of problems associated with alcohol consumption continues to be a high priority public health issue in spite of a certain drop in global consumption, in particular because drunkenness at weekends continues to be a frequently found form of consumption. There is sufficient research, at the present time, to endorse the various measures being applied, the reduction of easy access to its consumption being one of the most efficacious. But other methods have also been shown to be useful such as reducing the association between driving and drinking and controlling advertising. There are classic measures such as prevention among schoolchildren that require further research directed, most of all, at endorsing the results. And, there are new actions directed specifically at diminishing the effects of problems derived from alcohol consumption, finding alternatives to going out to have fun and drinking alcohol or to intervention in recreational contexts both from the cultural point of view and the physical context that could be promising but which also require adequate evaluation.

Key words: prevention, alcohol, taxes, prices, evaluation, review.

\section{INTRODUCCIÓN}

$\mathbf{E}$ alcohol es sin duda la sustancia de mayor penetración en la sociedad española como pone de manifiesto el hecho de que en 1999 el 87,1\% de la población de
15 a 65 años lo haya consumido 'alguna vez' en su vida; que un $74,6 \%$ lo hiciera durante 'los últimos 12 meses'; que un $61,7 \%$ lo hiciera 'en los últimos 30 días' y un 13,7\% 'bebiera a diario' en los últimos 12 meses"1. Por tanto, más allá de la alarma social existente alrededor de las drogas ilegales, tanto 
el alcohol como el tabaco son las drogas más usadas y las que generan con diferencia mayores problemas de salud pública en la actualidad. Pero el alcohol además de ser una droga es también un elemento cultural de primer orden en nuestra sociedad. Desde hace milenios muchas culturas han reservado al alcohol un lugar importante dentro de sus relaciones sociales e incluso rituales. En sociedades occidentales como la nuestra, donde la búsqueda del placer y del éxito social son elementos primordiales, donde el fin de semana y el tiempo libre en general alcanzan dimensiones casi míticas, el alcohol ocupa obviamente un lugar clave en este entramado, ejerciendo con autoridad su papel de rey de todas las drogas. Si bien hace unas décadas algunas de las drogas que se consumen ahora no se conocían o no tenían la popularidad de que gozan en estos momentos, su aparición no ha desplazado al alcohol sino que al contrario esta sustancia está presente en casi todas las combinaciones que hacen los jóvenes policonsumidores. Todas estas circunstancias hacen que la prevención de una droga legal, que es vista por una parte de la población como poseedora de virtudes positivas para la vida social y hasta saludable si se consume con moderación -como se insiste en muchos medios de comunicaciónno es una cuestión ni fácil de abordar ni fácil de resolver.

Hay aspectos relacionados con el consumo del alcohol y las circunstancias en que se produce que es esencial conocer y comprender en profundidad al emprender acciones preventivas. Se trata de cuestiones que pueden parecer demasiado generales pero que sirven para situar el problema en un momento dado, pues nos encontramos ante una situación dinámica donde hay muchos actores implicados. Nos referimos a cuestiones como la extensión y las pautas actuales de consumo, el papel de la edad y el género, el papel y la importancia de la industria alcoholera y de la industria de la noche, el contexto en el que se produce el consumo de alcohol, la cultura que se está generando alrededor de lo recreativo y el tiempo libre,..., que abordaremos a continuación y que son elementos a tener en cuenta para poder establecer una estrategia preventiva ligada a la magnitud y circunstancias del consumo y al contexto cultural y económico.

\subsection{Evolución y características del consu- mo.}

Es evidente que necesitamos saber a la hora de prevenir cual es la extensión del problema, a que grupos afecta especialmente, cuales son las formas de uso y abuso o cuales son las tendencias de este consumo. En el último informe del Observatorio español sobre Drogas se constata que "entre 1997 y 1999 el porcentaje de personas que han consumido alcohol alguna vez en su vida ha experimentado una ligera reducción que también se refleja en los indicadores de frecuencia anual y semanal y puede apreciarse un pequeño incremento en los indicadores de consumo mensual y diario'. La edad medida de inicio en el consumo de alcohol entre los hombres de 15 a 19 años en la encuesta domiciliaria del 97 era de 14,6 años (y las mujeres a los 15), mientras que en el 99 es a los 14,9 (y para las mujeres sigue a los 15).

Es decir que estaríamos ante un fenómeno que en los últimos años se estaría estabilizando en los aspectos cuantitativos. Sin embargo es "en los años 90 cuando ha saltado la alarma y la 'cuestión del alcohol' se ha convertido en una 'importante y dramática noticia' en realidad el consumo global de alcohol se mostraba cuando menos estable" ${ }^{2}$. Según estos mismos autores y otras fuentes ${ }^{3,4}, y$ atendiendo a los indicadores disponibles, no se puede hablar de que en los 90 exista un aumento del consumo de alcohol y de los problemas asociados. En general se acepta que el momento más alto de consumo per capita de la historia reciente fue el año 1.975 con 14,2 litros, y que dicha cantidad ha experimentado un descenso desde entonces. Los años 70 y la primera mitad de los 80 habrían sido los momentos de un mayor consumo entre la población general. 
Otra cuestión que debemos reseñar es la equiparación de formas de consumo entre los diversos países europeos ${ }^{4,5}$ y no sólo en lo referente a las cantidades totales que se consumen, sino al tipo de bebida (los países que consumían cerveza están incorporando el vino y viceversa) y a la forma de consumo (los países mediterráneos están desarrollando un consumo de fin de semana que sustituye al consumo diario ligado a las comidas que era más tradicional). Es importante por sus implicaciones preventivas observar cómo las evoluciones en los consumos han sido sorprendentemente similares en todos los países a pesar de las diferencias económicas y culturales, sin que se pueda pensar en un único factor causal en esta evolución (4). Estas modas que se extienden a través de las fronteras europeas o incluso más allá, pues existe una gran capacidad de asimilación cultural en el ámbito de la fiesta en estos momentos, llevan a plantear la necesidad de conocer mejor estos fenómenos y de tener la capacidad de intervenir en su generación para poder pensar en que la prevención tenga éxito en sus objetivos ${ }^{6}$.

\subsubsection{Pautas de consumo}

En las últimas décadas estamos asistiendo a cambios radicales en las formas de consumo del alcohol que se centran básicamente en el paso de un consumo diario a un consumo de fin de semana, lo cual ha sido más evidente en los países del sur de Europa. Ello se asocia además con una forma de beber más compulsiva, siendo entre los jóvenes donde son más evidentes estas nuevas formas de beber. Los consumidores diarios de alcohol siguen disminuyendo los últimos años en la población general pasando del $14,6 \%$ en la encuesta domiciliaria del 95 a $13,7 \%$ en la de 1999.

En una buena ilustración de lo que son las nuevas pautas de uso de drogas entre los jóvenes tenemos el estudio de la red europea de investigación Irefrea ${ }^{7}$ donde se entrevista a 1.340 jóvenes de cinco ciudades españolas y a 2.600 de 9 ciudades de otros tantos paí- ses europeos que salen de marcha en los lugares recreativos a los que acuden para divertirse -no se trata pues de muestras estadísticamente significativas, aunque sí aportan información que entendemos debe ser tenida en cuenta-. Vemos que sólo el $6,5 \%$ de los españoles y el $8,3 \%$ de los europeos consumen diariamente alcohol cuando solo el $7,5 \%$ de los españoles y el $13,2 \%$ de los europeos son abstemios o prácticamente no consumidores, lo cual lleva a la constatación que el grueso del consumo de alcohol es de fin de semana. Si es lógico esperar que exista un importante contingente de fumadores diarios de tabaco entre estos mismos jóvenes $(61,5 \%$ en la muestra española y $55 \%$ en la europea), menos conocido es el elevado número de consumidores diarios de cannabis $(15,8 \%$ entre los españoles y $10 \%$ entre los europeos). Algunos estudios sugieren que el consumo de alcohol y cannabis son sustitutivos o complementarios, ${ }^{8}$ en función de los precios, disponibilidad, ...

En algunas regiones de España donde ha existido la ocasión de hacer un seguimiento de los hábitos de consumo de los jóvenes ${ }^{10,11}$, se asistió a principios de los 90 a una polarización de los hábitos de consumo con un aumento importantísimo de los abstemios por un lado y los consumidores por el otro, con el afianzamiento de un grupo de consumidores de alto riesgo que Elzo ${ }^{11}$ sitúa en un $14,6 \%$ y Amengual ${ }^{10}$ eleva al $25 \%$ del conjunto, con un núcleo duro de un $5,7 \%$ del total. En población general y atendiendo únicamente a criterios de Unidades de bebida estándar consumidas a la semana la encuesta domiciliaria del 99 señala un 9,5\% de población de riesgo $(12,1 \%$ de varones y $6,9 \%$ de hembras).

Este beber de fin de semana de los jóvenes -junto con las prácticas de policonsumoobviamente tiene consecuencias distintas y plantea problemas preventivos distintos que en el caso del consumo diario más frecuente entre los adultos. Las características de estas nuevas formas de consumo de alcohol entre los jóvenes inciden en la patología observable. Hallamos menos patología orgánica entre 
este grupo de consumidores y aumentan los problemas conductuales derivados de este beber compulsivo. De hecho estas formas de consumo ya empiezan a tener su traducción en la demanda terapéutica de menores de 30 años que han pasado desde hace tiempo en algunos estudios del $15 \%$ al $24 \%^{12}$. Pero sobre todo es en las demandas de servicios de urgencia -sobre lo que hay pocos datos sistemáticos disponibles- donde sin lugar a dudas se tiene que reflejar más las consecuencias de estas formas de consumo.

Esta realidad del aumento del consumo juvenil y de los problemas asociados no nos debe impedir ver que el uso y el abuso del alcohol no es exclusivo de esta franja de edad y que, a pesar de que el grueso de los esfuerzos preventivos se dirigen a esta edad, no se debe de dejar de actuar en otras franjas de edad. En este sentido Monrás ${ }^{13}$ señala que en Cataluña la población atendida por problemas de alcoholismo es cada vez de mayor edad, especialmente en el caso de los varones. Estaría emergiendo, según el autor, un alcoholismo de 'tercera edad', lo cual supone que se debería incidir en la prevención de los factores de riesgo que estarían propulsando el aumento de la problemática en este sector de edad. Interesante también el dato de que en los datos de tratamiento en Cataluña no se estaría detectando un incremento del alcoholismo juvenil. ¿Inadecuación de la oferta terapéutica? ¿Falta de conciencia entre usuarios, familiares y profesionales sobre esta problemática? ¿Necesidad de campañas preventivas que faciliten que los jóvenes con problemas pidan ayuda?

\subsubsection{Embriagueces}

La embriaguez es el símbolo por excelencia de estas nuevas pautas de consumo: consumo de fin de semana en espacios recreativos. La necesidad de divertirse lleva a muchos jóvenes a beber compulsivamente muchas veces sin la intención de embriagarse -aunque éste acabe siendo el resultado bastantes veces- mientras que otros buscan directamente la embriaguez, que deja de ser fortuita. Pero también como sabemos la embriaguez es la expresión del alcoholismo crónico.

Al comparar la encuesta domiciliaria del 97 con la del 99 podemos constatar que han disminuido de manera sustancial las frecuencias más intensas de borracheras (diarias, semanales y mensuales) que del 5,3\% en 1997 se habría pasado en 1999 al 3,9\% todo ello referido a la población general. Pero la distribución de la problemática no es homogénea entre la población. Nuevamente nos encontramos con dos grupos: hay un grupo de adultos (entre los hombres de 30-34 años y los de 40-65 en la encuesta domiciliaria de 1999, mientras que en las mujeres es el grupo de 35-39 años aunque existe bastante diferencia porcentual con los hombres.) con borracheras diarias que seguramente corresponde al perfil clásico de alcohólico crónico. El otro grupo está formado por los jóvenes que salen con frecuencia los fines de semana y que se emborrachan con frecuencia durante sus salidas. Si acudimos nuevamente a los datos de Irefrea ${ }^{7}$, tenemos que sólo el $30,7 \%$ de los que salen los fines de semana no se habría emborrachado el último mes.

La embriaguez es un comportamiento que implica muchos riesgos (accidentes de tráfico, caídas, conducta violenta, relaciones sexuales sin precauciones o no deseadas,...) por lo que se convierte en la prioridad preventiva principal, especialmente en unos momentos en que los jóvenes españoles suelen emborracharse con frecuencia.

\subsection{Feminización de los consumos.}

Si bien el consumo diario de alcohol es un patrón marcadamente masculino $(21,1 \%$ de hombres y $6,2 \%$ de mujeres en 1999), sobre todo si nos referimos a la población de más de 40 años, debe destacarse el importante crecimiento que el consumo de alcohol ha registrado entre las mujeres. Entre las más jóvenes (de 15 a 19 años) en el periodo 19951999 el consumo de alcohol en el último mes que tiene una prevalencia de 37,7\% en 1995 
pasa a 50,9\% en 1999, que, por otro lado, es un porcentaje que se acerca al de los varones $54,9 \%{ }^{1}$. Ello lleva, desde el punto de vista preventivo, a la necesidad de entender los términos en que se está produciendo la evolución personal y social de la mujer, que lleva a un aumento espectacular en los consumos de alcohol y otras drogas como el tabaco, cannabis, etc, para ver que tipo de actuaciones se pueden emprender.

\subsection{País turístico y productor de alcohol. Importancia de la cultura recreativa.}

No es sin lugar a dudas una cuestión secundaria en cuanto al consumo real y a la representación social que se tiene del consumo y de los consumidores que España sea el tercer productor mundial de alcohol. A ello debemos añadir que somos un primer destino turístico lo cual tiene una influencia real en los hábitos de consumo así como en la disponibilidad de alcohol y locales de venta, precios, etc.

A la facilidad tradicional para la fiesta del pueblo español reflejada en la cantidad e importancia de eventos festivos en todas las ciudades del país se ha añadido todo el fenómeno turístico que afecta a gran parte de España lo cual ha supuesto profundizar en la práctica la accesibilidad al alcohol y aumentar las ocasiones de consumo, apoyado todo ello por una potentísima industria turística. Esta infraestructura poderosísima, sin rival en ningún lugar del mundo, apoya muy eficazmente una identificación cada vez mayor de gran parte de los jóvenes con una cultura recreativa de fin de semana, donde el alcohol y las otras drogas son un elemento imprescindible, y que se está imponiendo como una cultura hegemónica entre los jóvenes, donde realizan una parte importante de su socialización $^{6,7}$. Poder intervenir sobre esta dinámica cultural y económica es una cuestión de primer orden para la prevención. Hay que dejar de pensar en una prevención -a semejanza del tratamiento- basada en actuaciones puntuales más o menos sistemáticas para entrar en toda la dimensión cultural -y ética- que nos plantea la cultura recreativa.

\section{ESTRATEGIAS PREVENTIVAS}

Actuar preventivamente en el caso del alcoholismo parece que debería tratarse de: 1) definir el grupo sobre el que vamos a intervenir, 2) señalar unos objetivos prioritarios y 3) aplicar la estrategia que obtiene mejores resultados. Pero ya hemos visto, por lo que antecede, que señalar prioridades no es sencillo al ser el alcohol una droga legal, culturalmente muy arraigada e ideológicamente muy marcada. Si el acercamiento del problema del alcoholismo a la medicina fue en su momento un éxito importante, pues permitió su abordaje terapéutico sin las connotaciones morales y marginalizadoras al uso, en cambio ello no supuso unas ventajas similares para la prevención, pues en la concepción médica del alcoholismo los correlatos físicos y psiquiátricos han tenido un peso excesivo en la definición del conjunto. Ha sido la aparición de los estudios epidemiológicos los que han permitido resituar la visión clínica estereotipada. En efecto, los alcohólicos crónicos sólo representan una minoría dentro de los que tienen problemas alcohólicos ${ }^{14}$, por lo que es un error tener el 'alcoholismo crónico' como modelo de la prevención, especialmente en unos momentos en que, el cambio de pautas de consumo, hace que sea la embriaguez de fin de semana de los adolescentes y jóvenes el comportamiento por excelencia a prevenir.

“La gran inflexión en el mundo anglófono aceptando determinados puntos de vista de algunas publicaciones europeas y ciertos planteamientos de la OMS se produjo en 1980, al iniciarse el proceso de elaboración del DSM-III. Feighner y Spitzer dieron a la cuestión un giro copernicano para la psiquiatría convencional anglófona e incluso para determinadas teorizaciones de la Europa continental y para algunas 'escuelas' $u$ orientaciones psicológicas.... Incluyeron el alcoholismo entre los trastornos asociados o 
vinculados a la utilización de sustancias toxicomanígenas y no en la subcategoría de los trastornos de la personalidad"16. A partir de este momento la exposición al alcohol, como pasa en las otras drogodependencias pasa a ser un factor determinante dentro de la pluricausalidad del alcoholismo. "Por consiguiente, deben evaluarse como en el caso del tabaco o los opiáceos, la accesibilidad y la disponibilidad como factores de riesgo y modificar substancialmente los supuestos teóricos y prácticos de la prevención"15.

Paralelamente a estos esfuerzos de la medicina para situar el alcoholismo hay que hacer referencia a toda una tradición no médica del abordaje del alcoholismo que podemos situar alrededor de los movimientos de templanza, especialmente implantados en los países de base cultural protestante. La primera sociedad se funda en 1813 en Norteamérica, extendiéndose con rapidez dicho movimiento por Inglaterra y norte de Europa. En España "no faltaron informes, propuestas y teorizaciones sobre cómo combatir el alcoholismo en nuestro país, desde los planteamientos más pedagógicos como la educación de la infancia y la juventud o la pretensión de moralizar las costumbres de la población, hasta los más represivas como la supresión de las tabernas o la limitación de la libertad de funcionamiento de los despachos de bebidas alcohólicas que rápidamente chocaron con los intereses de los poderosos monopolios vitivinícolas españoles"16. Pero, citando literalmente a Piga y Marioni en su libro "Las bebidas alcohólicas. El alcoholismo", publicado en Barcelona en 1904: “Los gobiernos en su mayor parte, faltos del apoyo y del empuje de la opinión, no se deciden a abordar el problema con toda la tenacidad y energía que su importancia requiere. La masa general del público, ni se preocupa de la asistencia al alcoholismo, ni se interesa por las medidas que para combatirlo puedan adoptar aquellos, si no es para ponerlos en solfa o para protestar a grito pelado cuando vienen a herir una costumbre consagrada por la tradición o el hábito, uno de los mal entendidos derechos individuales o uno de los famosos intereses creados que van en contra de los intereses generales de la humanidad". Existe la mención de una de estas sociedades en Barcelona ${ }^{17}$. Mucho mejor documentada ${ }^{18}$ está la existencia de la 'Liga Antialcohólica Española' con sede en la provincia de Castellón, con su órgano de expresión 'El abstemio'. Dicha sociedad se crea oficialmente en 1911 y extiende irregularmente su actividad durante aquella década. Fue creada por Alfred Ecroyd, un comerciante inglés, y Miguel Gallart, un médico titular, en Artana pequeña localidad de Castellón.

\subsection{La paradoja preventiva.}

A la hora de seleccionar estrategias hace un tiempo los profesionales tuvieron que resolver la disyuntiva de si los esfuerzos deben concentrarse en poblaciones de alto riesgo o si se debe, por el contrario, optar por intervenir sobre la población general, pensando que de esta forma también se reducía el consumo y los problemas de los consumidores de riesgo. Si bien actuar sobre los consumidores de alto riesgo parece un objetivo obvio, diversos estudios han mostrado la mayor eficacia de aquellas estrategias que se dirigen a la población general, en lo que se ha denominado la paradoja preventiva ${ }^{19}$. La paradoja consistiría en que actuar sobre la población de alto riesgo, al tratarse en realidad de un sector mínimo de la población que consume, sólo prevendríamos una mínima parte de la problemática. La mayoría de la población, aunque individualmente son menos problemáticos, al ser mucho más numerosos son los que finalmente causan la mayor parte de los problemas.

Los movimientos de templanza, con toda su carga moral e ideológica, tenían ya entre sus prioridades el descenso del consumo global de la sociedad. Pero nos tenemos que remontar a 1946 para hallar constancia científica de la correlación entre consumo de alcohol per capita y mortalidad. Fue Lederman quien estudió los consumos y la mortalidad de diversas regiones de Francia y otros paí- 
ses llegando a mostrar una relación estrecha entre ambas circunstancias, que según él seguía leyes matemáticas. Muchos estudios han continuado esta línea de investigación poniendo en duda la relación matemática estricta pero confirmando la relación entre las variaciones del consumo global y la problemática generada por dicho consumo.

Por tanto si son los bebedores ocasionales y moderados los que cuantitativamente provocan más problemas, la prevención debería dirigirse sobre todo hacia ellos y ésta es la orientación preconizada por la Oficina Regional Europea de la $\mathrm{OMS}^{20}$ y el grupo de Edwards ${ }^{4}$. Algún estudio reciente que trata de comparar ambas políticas es menos concluyente entre los beneficios de una política sobre otra. Según Nörstrom ${ }^{21}$ (1995) una disminución en un $25 \%$ del consumo per capita reduciría la mortalidad inducida por el alcohol debido a la cirrosis, accidentes y suicidios en 58,36 y 38 por cien respectivamente. Para conseguir un impacto similar con las estrategias centradas en el alto riesgo sería necesario que el consumo de las grandes bebedores (el $5 \%$ del total) se vea reducido en un $36 \%$. Otros datos importantes de este estudio consisten en mostrar como las estrategias que se focalizan en las poblaciones de riesgo darán mejores resultados para las patologías hepáticas (y probablemente para otras patologías más severas), mientras la estrategia poblacional también tiene un impacto sustancial en este contexto, aun cuando muestra más su potencial es en relación con accidentes y suicidios.

En todo caso, estamos ante dos estrategias perfectamente válidas. Quizás nos interesa aquí subrayar la importancia de las actividades preventivas en población general pues son las que desde la perspectiva médica pueden parecen menos evidentes. Pero, la cuestión crucial consiste entonces en saber qué estrategia es más practicable. Posiblemente una combinación de las dos estrategias sea la postura más inteligente, pues con frecuencia se ha mostrado la importancia de la sinergia entre las actividades preventivas. Es importante señalar que si de 1992 a 1999 el
Plan de Acción Europeo sobre el Alcohol2 apoyaba las actuaciones basadas en la actuaciones dirigidas a la población general, en el nuevo período 2000-2005 se apoyan preferentemente las estrategias basadas en actuaciones sobre situaciones de riesgo ${ }^{23}$. Esto tiene que ver seguramente con que las políticas referidas a las situaciones de riesgo son más fácilmente aplicadas que las que se dirigen a la disminución del consumo en la población general -de hecho los impuestos han bajado en un tercio de los países europeos- $y$ esto puede tener repercusiones negativas a medio plazo, especialmente cuando la producción de vino, por ejemplo, ha aumentado en el periodo 1995-97 un 9,4\% ${ }^{24}$.

\section{INTERVENCIONES.}

\subsection{Disminuir la accesibilidad al alcohol.}

Todos los enfoques dirigidos al control del consumo tienen influencia en la disminución de dicho consumo ${ }^{25}$. Bajo este epígrafe caben actuaciones muy diversas, como la famosa ley seca aplicada en USA hasta el control de la venta a menores. Disminuir la accesibilidad sigue siendo una de las medidas que más claramente tienen una influencia en el consumo y en los problemas relacionados con dicho consumo, lo cual, más allá de las evidentes dificultades políticas para su puesta en marcha, viene avalado por una suficiente base experimental, sin que sea válido el argumento de que la población siempre será capaz de conseguir alcohol, especialmente los bebedores excesivos a la luz de las numerosas investigaciones realizadas ${ }^{4}$. La investigación actual avala suficientemente no sólo que las medidas de control afectan a los consumidores de riesgo sino que incluso son los más afectados por dichas medidas ${ }^{26}$.

Diversos estudios ${ }^{4}$ avalan que todo aumento en la disponibilidad del vino conlleva un aumento neto del consumo de alcohol, aunque llegue a producirse algún tipo de sustitución, de la misma forma que la introducción 
de cerveza de media y alta graduación en países como Suecia, Noruega y Finlandia se acompañó de un incremento en el consumo de este producto. La autorización a vender cerveza en las tiendas en Finlandia supuso al cabo de un año un aumento del consumo del $46 \%$ y un aumento de las muertes relacionadas con el consumo de alcohol del $58 \%{ }^{26}$. Entre 1985 y 1987, durante el mandato de Gorbatchov, hubo en Rusia una campaña antialcohol ${ }^{27}$ cuyos elementos centrales fueron poner en marcha restricciones en la producción y venta de bebidas alcohólicas, el control de los precios y la prohibición de anunciarse. De acuerdo con el Comité Nacional de Estadísticas de Rusia se experimentó una caída brusca en el consumo de 10,4 litros de alcohol puro en 1984 a 3,9 en 1987. Los autores admiten que las dificultades en el suministro oficial venían compensadas en parte por obtener alcohol en el mercado negro, pero aún así consideran que se produjo una caída en el consumo. En todo caso, otras fuentes estadísticas avalaban también el éxito en términos de salud pública de dichas medidas: una reducción en un $26 \%$ de acciones criminales producidas bajo el efecto de la intoxicación alcohólica y una reducción en el absentismo laboral debido al alcohol del $30 \%$. La reducción del número de muertes relacionados con el alcohol durante este período de 1984-87 fue del $54 \%{ }^{26}$.

La adopción de este tipo de medidas choca sin lugar a dudas con intereses económicos y pueden fácilmente levantar la protesta de diversos sectores, pero ello no invalida que la medida ha demostrado su eficacia en diversos contextos culturales. Pero no son sólo los industriales afectados los que se oponen a estas medidas en España, sino que hasta la misma población puede reaccionar negativamente -a falta de una adecuada información y mentalización- a las medidas de control lo que lleva a las administraciones a ser reticentes a tomar dicho tipo de medidas. La publicidad que tienen los accidentes de jóvenes de fin de semana y el conflicto de intereses que genera la actividad recreativa nocturna en el vecindario esta generando una sensibilización favorable a este tipo de medidas. Una de las medidas que no se aplican muchas veces en nuestro país con el rigor necesario es la entrada de adolescentes de menos de 16 años en locales donde tienen prohibida la entrada por ley al haber consumo de alcohol y el incumplimiento sistemático de horarios de cierre de bares, discotecas y otros locales por la noche.

\subsection{La influencia del precio de las bebidas alcohólicas.}

El gasto en alcohol es un capítulo importante de la economía de los jóvenes. Según el estudio de Irefrea sobre jóvenes que salen de marcha $^{7}$ de las 9.915 pesetas que se gastan como media cada semana 2.302 se destinarían a bebidas alcohólicas y 2.556 a la compra de drogas ilegales. Por supuesto es también un apartado importante en la economía de los adultos, por lo que no tiene que extrañar que las variaciones de los precios de las bebidas repercutan en el uso de alcohol. De hecho el fenómeno del 'botellón' (jóvenes con sus vehículos que se reúnen en espacios públicos para consumir alcohol antes de ir a los bares o discos), tan popular desde hace unos años en muchas ciudades españolas, nace como un intento de beber a precios de coste de la bebida.

La revisión hecha por Edwards ${ }^{4}$ sobre el tema es concluyente: "de todos los factores interactivos, el coste de las bebidas alcohólicas es uno de los métodos más eficaces para disminuir tanto la cantidad total de alcohol consumido como el número de bebidas que una persona toma....Si no varían otros factores, el consumo de alcohol por una población siempre dependerá, en mayor o menor grado, del precio de las bebidas alcohólicas. Tanto el consumo de los grandes bebedores como el de los pequeños consumidores depende del precio del alcohol".

Si bien las diversas revisiones coinciden en la relación más precio menos consumo, la magnitud del efecto es a veces difícil de precisar dependiendo de factores tales como el 
tipo de bebida, tipo de consumo, el país, el momento, genero, los ingresos personales,... Podría pensarse que estas medidas pueden ser más efectivas en función de la capacidad económica del sujeto, por lo que es posible que estas estrategias sean más eficaces en los jóvenes. En efecto algunas investigaciones apuntan en esta dirección. El aumento del precio de la cerveza a través de impuestos reduce la cantidad y la frecuencia de su consumo entre los jóvenes de 16 a 21 años, siendo mayor este efecto entre los bebedores habituales o bastante habituales que entre los bebedores esporádicos ${ }^{28,29}$. Kenkel ${ }^{30}$ también encuentra que el consumo abusivo de alcohol de jóvenes y adolescentes es más sensible a los precios que el de los adultos en condiciones similares, siendo ello todavía más cierto en el caso de las chicas. También parece que el aumento de precios es todavía más eficaz si los precios ya son altos según la experiencia de los países escandinavos.

Se puede suponer que la capacidad económica personal tiene que ser un factor a tener en cuenta a la hora de estudiar los efectos del aumento de precios, sin embargo es una cuestión poco estudiada aunque la evidencia parece apuntar que si ejerce un papel importante ${ }^{31}$.

Estamos pues ante una estrategia que para las administraciones supone poco gasto directo y que ha mostrado su eficacia preventiva, por lo que consideramos que una firme política impositiva debe formar parte de toda estrategia preventiva del uso indebido del alcohol.

\subsection{Clase social.}

Es esta una cuestión poco estudiada normalmente pero de indudables implicaciones prácticas para la prevención tal como veremos. Consumir alcohol, fumar, hacer ejercicio físico o la obesidad dependen de estilos de vida que se han mostrado muy relacionados con la clase social del individuo. Es muy interesante ver que en una revisión de las Encuestas Nacionales de Salud españolas de
1987 a 1997 los grupos sociales menos favorecidos (IV y V) presentaban al final del periodo analizado incluso peores índices en la adopción de hábitos saludables. Por el contrario los grupos sociales más favorecidos (I, II, III) mostraban una ganancia neta en la adquisición de hábitos más favorables ${ }^{32}$.

En los estudios de Irefrea ${ }^{6,7,33}$ se comprueba como una gran parte de la población juvenil que sale los fines de semana pertenece a clases medias o media-altas. Son estos grupo sociales -entre otras cosas por que salir los fines de semana cuesta dinero- los que imponen las modas y las formas de consumo, sin embargo los consumos más altos o problemáticos recaen más -aunque no únicamente- en las clases sociales menos pudientes económicamente.

El significado de estas cuestiones necesita claramente mayor investigación, pero las políticas preventivas deberían tener en cuenta esta dimensión.

\subsection{Beber y conducir.}

La relación entre problemas para conducir y beber alcohol es algo firmemente establecido por la investigación. Una revisión de 112 estudios concluye que habilidades requeridas para la conducción se deterioran incluso a partir de niveles de alcohol en sangres míni$\operatorname{mos}^{34}$. La presencia de alcohol en sangre afecta más a los menores de 21 años según diversos estudios, siendo el riesgo relativo de estar implicado en un accidente mortal en USA para alcoholemias entre 0,8 y 1 del $11 \%$ para conductores de 35 o más años y del $52 \%$ para conductores varones entre 16 y 20 años ${ }^{35}$.

La variedad de situaciones legales existente en los diversos estados norteamericanos permite estudiar los efectos de diversas medidas legales. La situación actual es que durante la última década en USA han decrecido el número de conductores con alcoholemias positivas durante los fines de semana, así como el número de accidentes de tráfico mortales relacionados con el consumo de 
alcohol $^{36}$. Existen Estados de Norteamérica que aplican leyes de 'tolerancia cero' (0,02\% de alcohol o menos en sangre) para menores de 21 años; de los 14 estados que han reducido el limite legal de alcoholemia entre la población general demuestran descensos considerables de hasta un $20 \%$ en comparación con aquellos estados que no aplican la misma normativa ${ }^{37,38}$. Un estudio a nivel nacional revela que los estados con un límite de alcoholemia de 0,8 para los mayores de 21 años -hay 27 estados en la actualidad con dicho limite legal en USA- tienen menores proporciones de adultos implicados en accidentes mortales que los estados que toleran una alcoholemia mayor ${ }^{39}$.

Existen medidas legales que resulta imposible imaginar que puedan ser aplicadas en nuestro contexto cultural como prohibir el acceso al alcohol a menores de 21 años, aunque es esta una medida legal vigente en USA. Diversos estudios demuestran como el aumento de la edad legal mínima para beber en USA se ha acompañado de una disminución en el consumo de alcohol, de menos accidentes de tráfico y, consiguientemente, de menos víctimas relacionadas con estos accidentes entre menores de 21 años $^{40}$, de la misma forma que el descenso de 21 a 18 años que se produjo en diversos estados durante el año 1979 supuso un aumento de accidentes (Vingilis y DeGenova, 1984, citado por Allen ${ }^{41}$.

La retirada del carnet de conducir sí que es una medida que se aplica en España para conductores con alcoholemias positivas. Esta medida se ha mostrado eficaz en USA para reducir tanto los accidentes mortales relacionados con el alcohol $(28,37 \%)$ como futuras sanciones por conducir habiendo bebido ${ }^{42}$, aunque existe evidencia de que el $75 \%$ de conductores sancionados en USA siguen conduciendo. Hay sistemas que bloquean la puesta en marcha del motor mediante sistemas que detectan el alcohol en el aliento, lo cual se utiliza como una alternativa a la suspensión del carnet de conducir en algunos lugares de USA.
El simple hecho de que se realicen alcoholemias frecuentemente pensamos que de por si ya es un elemento preventivo. En España se realizaron 1.762 .000 controles de alcoholemia en el 2000, casi 250.000 más que en 1999, resultando un $5 \%$ positivos, o sea 88.000 conductores que conducían habiendo bebido de más según datos de la Dirección General de Tráfico.

La existencia de investigación suficiente en este campo (ver Plasencia, 2002 en esta monografía) y la implicación de los medios de comunicación ha permitido que éste se convierta en uno de los temas estrella a la hora de sensibilizar y plantear la prevención del alcoholismo y de los problemas asociados. La existencia de una base experimental y el apoyo de la opinión pública permite desde hace años el desarrollo de variadas estrategias preventivas de diversa índole: desde sanciones ligadas a la conducción bajo los efectos del alcohol hasta medidas preventivas inspiradas en la reducción del daño como las que propician que cuando salen un grupo de amigos uno de ellos permanezca sobrio para poder conducir a la vuelta.

\subsection{La publicidad de bebidas alcohólicas.}

La publicidad se está convirtiendo en una cuestión de una importancia clave. Las alcoholeras han tendido a organizarse durante los últimos años en grupos de presión (Grupo Ámsterdam, Portman, etc) importantísimos y muy eficaces en la defensa de sus intereses. Alertados por lo que ha ocurrido con la industria tabacalera, han aprendido la lección y su publicidad no va dirigida únicamente a promocionar tal o cual producto sino a 'informar' y convencer a la opinión pública de las bondades de las bebidas alcohólicas. De una forma sutil-difícilmente detectable para el ciudadano- filtran y potencian noticias relacionadas con los supuestos efectos saludables del alcohol. Las noticias en este sentido reciben una amplísima cobertura en los medios de comunicación. Son muchos los esfuerzos económicos en mantener a una serie de 
periodistas especializados que se encargan de presentar con la faceta adecuada y con la insistencia suficiente este tipo de noticias.

Otro punto importante dentro de esta estrategia consiste en buscar el apoyo -y evitar el rechazo- de grupos de profesionales, en especial el grupo sanitario. En algunas revistas dependientes de instituciones médicas en nuestro país han aparecido durante los últimos años secciones donde se hace una oferta de vinos, se instruye al médico para que sea un experto enólogo, etc. Las alcoholeras han entendido perfectamente que el consumo de alcohol es un hecho básicamente cultural y por tanto quieren estar presentes en la definición que nuestra sociedad hace del consumo de alcohol. En definitiva se trata de evitar por todos los medios que el alcohol sea demonizado y mostrar la cara amable de las alcoholeras dispuestas a colaborar para evitar problemas de abuso. En otros países se produce en ocasiones la colaboración entre sociedades científicas y de profesionales con las alcoholeras para la organización de eventos o de investigaciones, cuestión que no parece darse en nuestro país.

¿Y que pasa con la publicidad directa de bebidas alcohólicas? El sentido común nos lleva a pensar que si la publicidad funciona para la promoción de los más diversos productos, ¿porqué no iba a funcionar la publicidad en la promoción de las bebidas alcohólicas como pretenden los grupos organizados de alcoholeras si la publicidad funciona para promocionar los más diversos productos? ¿Acaso las empresas gastan en publicidad por una simple cuestión narcisista sin pretender aumentar sus ventas? Según estos grupos que representan los intereses de los empresas alcoholeras, la publicidad no aumentaría el consumo sino que simplemente influiría en la elección de un tipo de bebidas frente a otras, sin influir en la cantidad total consumida. En efecto, hasta ahora había sido difícil de demostrar la relación entre publicidad y consumo. Smart ${ }^{43}$ estudia los efectos a corto (14 meses) y largo plazo de la prohibición de la publicidad sobre alcohol en los medios de comunicación de British Columbia, Manitoba, Noruega y Finlandia sin encontrar diferencias en los consumos anteriores y posteriores a la prohibición. Pero, una vez corregidos problemas metodológicos actualmente se dispone de datos que sugieren que la publicidad tiene un impacto pequeño, pero significativo, sobre los hábitos alcohólicos (4). En cambio mucho más eficaz parece ser la prohibición de publicidad sobre cigarrillos en los medios impresos a partir de un seguimiento hecho en New Zealand durante 13 años $^{44}$, lo cual nos llevaría a pensar en la importancia de la representación social que tiene la sociedad de cada una de estas dos drogas. Sabemos que muchos niños hasta los diez años mantienen una postura anti alcohol -y anti tabaco- pero que paulatinamente esta opinión va cambiando hacia posiciones más favorables hacia el consumo de alcohol influidos por los padres, los amigos y los medios de comunicación y publicidad $^{45}$.

En nuestro existen algunas limitaciones a la publicidad como la de prohibir publicidad de bebidas alcohólicas de alta graduación en $T V$, pero claramente estas medidas son insuficientes -y muchas veces se hace además un seguimiento inadecuado, aunque hay que decir que las resistencias a aumentar la presión en este campo también son frecuentes en otros países europeos. Problemas asociados son los de la esponsorización de eventos culturales o deportivos que muchas veces dependen de la industria alcoholera, que encuentra así una forma de burlar controles.

\subsection{Las campañas de prevención en po- blación general}

Entre las medidas más conocidas por la población general, y que ella misma es la destinataria, son las campañas contra el alcohol mediante publicidad y la utilización de los medios de comunicación. La gran verdad es que una ínfima parte de estas actuaciones ha sido objeto de evaluación. Cabe la justificación de que la evaluación de estas campañas 
supone un gran reto metodológico y de que por otra parte no se destinan recursos para que estas evaluaciones se realicen. El hecho es que muchas de estas campañas responden en todos los países a intereses muy inmediatos de las instituciones más que a verdaderas estrategias preventivas ${ }^{46}$. Más que esperar efectos inmediatos sobre los consumos a partir de las campañas - hecho que ha sido difícil de demostrar hasta ahora -, lo que debemos esperar es un aumento del interés de la población hacia estos temas, lo cual no deja de ser importante. Pensamos que su asociación a otro tipo de medidas preventivas puede crear una sinergia muy interesante.

Una forma interesante de hacer campaña es la de facilitar la creación de mensajes periodísticos en relación con accidentes, descubrimientos, etc. "Como ha sido demostrado por numerosos estudios, los mensajes en los medios de comunicación sobre seguridad y salud - cuando están bien diseñados - pueden tener una influencia considerable sobre la información, las actitudes y el comportamiento del público general. No obstante, el hecho real es que dichos mensajes tienen que competir en atraer la atención de muchos otros contenidos de los medios de comunicación y puede ocurrir, por consiguiente, que atraigan poco la atención" ${ }^{47}$. En España las campañas ligadas a la conducción son los que más se prodigan y en el recuerdo está una campaña de los años setenta "Siempre con una copa menos", siendo actualmente los mensajes de dichas campañas cada vez más asociados a los problemas que acarrea la asociación beber / conducir. Los mensajes de salud que utilizan el miedo deben cumplir dos condiciones para que sirvan: provocar la atención del sujeto $-y$ no sólo miedo- e ir acompañados de una recomendación percibida como eficaz para eliminar la amenaza ${ }^{48}$.

\subsection{Programas escolares.}

Estamos ante una de las medidas preferidas por la población española y que gozan de más consenso. Existe una larga tradición en nuestro país de implementación de programas escolares contra el alcohol y contra el resto de drogas ${ }^{49}$. La calidad media de los programas es seguramente más alta pero sigue sin resolverse -también en el resto de Europa y en otros lugares- si los programas son realmente efectivos. Ha aumentado la evaluación del proceso de muchos programas pero sigue sin haber una evaluación de los resultados, sin lo cual es difícil de afirmar si un programa funciona o no. Esta situación podía excusarse hace unos años pero hoy en día es ya difícil de explicar que no se progrese en dicha situación y se avance hacia una prevención institucionalizada y científica ${ }^{50}$. El tiempo corre en nuestra contra, y dado que estas cuestiones están planteadas desde hace tiempo, si no se resuelven cabe esperar que se resienta el prestigio de la prevención.

En efecto las cosas no pintan en general muy positivas. La última revisión de Foxcroft $^{51}$, que amplia las dos anteriores de 1995 y 1997 y que se concentra exclusivamente en los resultados -es decir si como consecuencia de los programas la gente bebía menos o tenía menos problemas con su consumo-, se analizan unos 6000 títulos a partir de búsquedas bibliográficas, que acaban convirtiéndose en sólo 196 documentos que merece la pena analizar, quedando finalmente 56 estudios -46 referentes a programas escolares- que reunieran los criterios científicos que permitieran sacar conclusiones sobre ellos. La mayor parte de los estudios eran americanos -sólo 2 británicos y 1 sueco como estudios europeos- seguramente porque son los que más investigan y además por el sesgo que supone la lengua inglesa en las búsquedas bibliográficas. Sólo 3 estudios de todo el conjunto han demostrado eficacia después de 3 años entre ellos un programa escolar de Botvin conocido entre nosotros por la adaptación de su programa a España en un programa llamado 'Construyendo salud' con el apoyo del Plan Nacional sobre Drogas, y los Ministerios de Sanidad y de Educación y Ciencia ${ }^{52}$. Precisamente éste último programa no ha sido incluido en esta revisión ni tampoco la 
evaluación del 'Tu decides' programa que se viene aplicando en Mallorca y Barcelona y que cuenta con dos evaluaciones en condiciones experimentales habiendo mostrado su eficacia entre los jóvenes incluso después de dos años de aplicación del programa ${ }^{53}$, por no estar sus evaluaciones en bases de datos en lengua inglesa. La situación no puede ser por tanto más deprimente nos guste o no y las instituciones y profesionales deben reaccionar con coherencia y firmeza ante esta situación.

Esta resistencia a evaluar y a utilizar programas evaluados tiene diversas causas, más allá de la obvia de que se requiere tiempo, dedicación y dinero. Nancy Tobler ${ }^{54}$ autora de dos metanálisis en 1986 y 1993 de programas preventivos opina que por un lado no se estaría haciendo una promoción suficiente y eficaz de los programas que han sido evaluados. Por otro lado, los programas interactivos - que requieren una pedagogía activa por parte del profesorado en el caso de los programas escolares- que serían significativamente los más eficaces, de acuerdo con el metanálisis realizado, son también más difíciles y caros de aplicar. Podemos, además, entender las implicaciones políticas y profesionales que existen en aceptar que lo que se viene haciendo no sirve.

Por tanto parece ineludible plantearse seriamente la evaluación de resultados sobre todo cuando son programas apoyados por instituciones potentes. La situación ideal sería seguramente mucha menos variedad de programas y que los programas que se utilicen estén evaluados en sus resultados -aunque también hay que complementar dicha evaluación con otras que tengan en cuenta los aspectos prácticos y de generalización de un programa.

En una evaluación del coste-eficacia de diversos programas escolares -en este caso se trataba de programas de cocaína- a través de un programa matemático de simulación se concluía que los beneficios de un programa eficaz pueden deberse a efectos indirectos como el impacto que el programa tiene sobre amigos y conocidos ${ }^{55}$.

\subsection{Programas comunitarios}

Comentarios similares a los de la prevención escolar podemos realizar para los programas comunitarios, con el agravante de que, por su misma naturaleza, son todavía más difíciles de definir, de delimitar y evaluar. Las posibilidades de intervención que ofrece este abordaje son prácticamente infinitas. Esta gran versatilidad lleva a que existan modelos menos definidos y comparables, tanto a nivel europe $0^{56}$ como norteamericano $^{57}$. Los programas que consiguen mejores resultados son los que se asocian con alguna situación concreta sobre la que la sociedad está concienciada como es la conducción de vehículos. Los programas comunitarios son importantes pues de ellos depende básicamente los cambios de opinión en la población que permiten adoptar políticas de control sobre la oferta con garantía de éxito. Por otra parte cabe pensar, de acuerdo a los modelos explicativos del uso y abuso de alcohol y otras drogas $^{57}$, que los programas en los que interviene la comunidad tienen que ser mucho más potentes en sus resultados.

En la revisión que hace Foxcroft ${ }^{51}$ sobre programas preventivos eficaces encuentra un programa comunitario ${ }^{57,59}$ que ha demostrado un $10 \%$ de reducción en accidentes de trafico relacionados con el alcohol teniendo en cuenta todos los conductores (no sólo los jóvenes) y encontró también que disminuían los lugares que vendían alcohol a menores bajo la edad legal (que recordemos que en USA es de 21 años en muchos estados). En esta misma revisión hay otro programa que en este caso se basa en prevención familiar ${ }^{60,}$ ${ }^{61}$ se muestra muy eficaz en la prevención de varias conductas relacionadas con el alcohol, con la particularidad de que la eficacia aumentaba a medida que pasaba el tiempo.

En otra investigación se considera que mejorar el entorno físico y social puede ser una medida eficaz y con una buena relación coste/beneficio como son ambientes de alto riesgo para el abuso de alcohol como los campus universitarios ${ }^{62}$. 
Una forma de prevención comunitaria que en España se ha desarrollado con gran potencia los últimos años son las iniciativas municipales que organizan por la noche de los fines de semana actividades alternativas en los centros deportivos y centros culturales. Estas iniciativas surgen hace unos años en Gijón con la denominación de 'Abierto hasta el amanecer' y se han extendido ya por más de veinte ciudades españolas. En el programa madrileño ('La noche más joven') que va desde las 22,30 hasta las 2,30 han participado durante este último año alrededor de 30.000 personas, siendo la mayoría de más de 20 años, pero dicen los organizadores que a quienes quieren captar especialmente es a los adolescentes de 12 a 16 años. Siendo iniciativas muy interesantes por lo que suponen de apoyar una cultura alternativa donde el consumo de sustancias deja de ser el centro, no existe una evaluación de estas actividades preventivas, lo cual es preocupante pues se están extendiendo por todo el país y no se puede presuponer automáticamente cuales van a ser sus resultados y que no tengan efectos contrapreventivos. Pensamos que son muchas cosas las que se tienen que controlar en esta evaluación, como, por ejemplo, los efectos que puede tener sobre pre-adolescentes a los que se les 'facilita' salir de marcha por la noche desde tan temprana edad. Al parecer en lo que tienen menos éxito es en rescatar jóvenes de alto riesgo o que ya estén haciendo consumos de alcohol y drogas importantes, razones sin embargo que han sido aducidas para poner en marcha este tipo de programas.

\subsection{Intervenciones en los Servicios de Ur- gencias.}

Existen experiencias evaluadas de la eficacia de intervenciones aprovechando la mayor sensibilización de las personas accidentadas que acuden a un servicio de urgencias. Dichas intervenciones muestran ser eficaces en reducir las readmisiones por accidentes relacionados con beber y conducir ${ }^{63}$, así como una menor frecuencia en beber y conducir, multas de tráfico, accidentes y otros problemas relacionados con el alcohol entre los conductores de 18 y 19 años ${ }^{64}$. Entre nosotros ${ }^{65}$ ya se están poniendo en marcha experiencias similares.

Se ha probado también la eficacia de sesiones con personas que han sufrido accidentes o que han perdido a algún familiar en un accidente que relatan el impacto que dichos eventos han tenido en sus vidas. Los resultados de las evaluaciones son contradictorios ${ }^{66}$.

\subsection{Intervenciones sobre el contexto re- creativo y los lugares de consumo}

Se trata sin lugar a dudas de una línea de intervenciones muy prometedoras. Se han desarrollado particularmente a partir de la necesidad, especialmente en el Reino Unido, de controlar en términos de salud pública los raves, eventos ilegales muy ligados a la cultura techno, con altos consumos de 'drogas de diseño' y por supuesto de alcohol. Ante la inevitabilidad de los hechos se opta por una línea de colaboración con los organizadores de dichas fiestas. Se pacta con ellos una serie de medidas como la accesibilidad al agua, la disponibilidad de dispensadores de preservativos, etc. Se trata en definitiva de intervenir directamente sobre los problemas que se generan a partir del consumo excesivo de alcohol y otras drogas (violencia, caídas, relaciones sexuales sin protección, heridas con los cristales de los vasos, ...). En 1996 el London Drug Policy Forum publica una serie de normas "Safer Dancing Guidelines" que incluye además de los puntos anteriores la formación de porteros, camareros y el resto del staff, cuidar el diseño del local, medios y formación en primeros auxilios, existencia de transporte,... Se han hecho muchos progresos en este sentido en el Reino Unido -también en Holanda, Alemania,...- especialmente en las grandes discotecas y orientaciones como Safer Dance o Club Health ${ }^{67}$ son populares. En Italia la asociación de empresarios de discotecas está colaborando actualmente -a partir de la muerte de 
varios jóvenes por consumo de éxtasis- a diversos niveles con la administración y se están elaborando una serie de normas legales que regule el funcionamiento de las discotecas.

Lo importante de estas soluciones es que, en algunos países por lo menos, van relativamente a favor de corriente. Estas medidas pueden funcionar sin tener que convencer a las personas para que beban menos, pues se concentran en las consecuencias de estas conductas, a través de crear situaciones más favorables. Al crearse un consenso sobre este tipo de medidas se hace posible avanzar hacia otro tipo de providencias que cuestionen el contexto recreativo hegemónico en el que estamos inmersos. Hasta ahora todas las medidas eran recibidas como represivas, mientras que a partir de estas orientaciones se hace posible pensar y pactar soluciones validas para los clientes, los empresarios y la administración. Entendemos que este tipo de soluciones no deben entenderse como simples medidas de reducción del daño -en cuyo caso aunque beneficiosas a corto plazo, servirían para perpetuar un único sistema de entender la diversión- sino que además deben servir como plataforma para generar un contexto recreativo más diverso, donde el consumo de alcohol y drogas no sean cuestiones tan centrales ${ }^{68}$.

\section{CONCLUSIONES}

Durante los últimos años se ha producido un descenso en el consumo absoluto de alcohol entre la población, lo cual es una buena noticia. No obstante también las últimos décadas han visto la expansión entre los jóvenes de formas de beber los fines de semana que conducen a la embriaguez frecuente, lo cual conlleva además importantes problemas asociados. La prevención de esta forma de beber debe ser el centro de la preocupación sin olvidar obviamente la prevención de otras formas de consumo más clásicas.
Son muy variadas las actividades preventivas que se han incluido en este esta revisión. Nos encontramos con intervenciones legislativas, otras están relacionadas con los impuestos o el control de los precios, en otros casos son programas escolares en los que se aborda el problema del alcohol de forma separada o conjunta con el resto de drogas, otros programas intentan intervenir sobre el contexto físico o cultural en los que se desarrolla la actividad recreativa,... ¿Qué programas se deben priorizar? ¿Por qué se aplican más unos programas que otros? ¿Quién decide estas prioridades? En relación con esta cuestión Room ${ }^{26}$ establece una interesante comparación entre las estrategias que son más eficaces (impuestos, disminuir su disponibilidad, algunas medidas de reducción del daño y disuasión especialmente en el contexto de beber y conducir) y las más populares (programas educacionales especialmente en la escuela, facilitar alternativas a beber, tratamiento y disuasión en beber y conducir). A excepción de la disuasión sobre beber y conducir no hay coincidencia en el resto. Según él la explicación a esta falta de coincidencia es que las medidas eficaces, por un lado, afectan intereses económicos y, por el otro, entran en conflicto con valores e ideologías de la sociedad o del espíritu de los tiempos. Claramente las medidas más populares -con excepción del beber y conducirsuponen poca implicación por parte de los adultos y la sociedad. Se traslada el problema para que lo resuelvan a los que supuestamente lo pueden tener (los jóvenes para lo que se les educa) o a los que lo sufren ya (a través del tratamiento), pero cuesta -dentro de la ambivalencia típica de la sociedad hacia sus drogas legales- adoptar otro tipo de medidas más intervensionistas.

Como sociedad nos sentimos más cómodos con estrategias como los programas escolares -y últimamente con la búsqueda de alternativas a salir de marcha en los centros deportivos sin ofrecer alcohol- que con medidas más restrictivas, como las políticas legislativas o impositivas -y su cumplimiento eficaz- dirigidas a dificultar el acceso al alco- 
hol. Estas últimas medidas requieren un mayor consenso social y posiblemente la población española sigue sin conocer y valorar adecuadamente los problemas que se derivan del consumo de alcohol y de determinadas formas de hacer fiesta. Edwards ${ }^{4}$, seguramente más identificado con políticas de control social, es bastante crítico con las ventajas de este tipo de intervenciones como la prevención escolar, por otro lado muy demandadas por la población. Pero hasta en nuestro país -pensemos que fenómenos como el del 'botellón' son totalmente inéditos en nuestros países vecinos- la tradicional tolerancia hacia la fiesta y el consumo callejero de alcohol tiene un límite y las protestas vecinales están influyendo en el ánimo de los legisladores para que se refuercen las posibilidades legales de actuación. Recientemente la Cámara autonómica andaluza se hace eco de las demandas vecinales y cede potestad a los ayuntamientos para que puedan actuar con más facilidad. Alguna otra autonomía tomó hace algún tiempo medidas similares. Pero ¿qué impidió actuar hace unos años, cuando el movimiento era todavía incipiente y que hubiese sido mucho más fácil reconducir la situación? ¿Cuan eficaces y con que diligencia se van a aplicar medidas correctoras?

Pero además de estas estrategias entendemos que donde hay que estar especialmente atentos es sobre todo el desarrollo cultural ligado con lo recreativo. Existe un importantísimo desarrollo cultural en este sentido animado por la industria del tiempo libre, por la mayor disponibilidad económica de los jóvenes y por el menor control social y familiar que se ejerce sobre ellos, junto con nuevas formas de entender la vida más individualistas y hedonistas. En la práctica esta cultura recreativa tiende a asociarse excesivamente con el consumo de alcohol y otras drogas, como instrumentos privilegiados para conseguir la diversión. Es importante por ello desde el punto de vista preventivo entender mejor como se construye esta cultura $y$, en segundo lugar, aprender de que forma podemos ser elementos activos en esta construcción.
La prevención que tenemos y que vamos a tener depende de los políticos o responsables de las políticas sociales y económicas, de los profesionales y también de la población destinataria. Cada uno de ellos tiene su responsabilidad que en ocasiones se solapa con la de los demás y a veces no. Es evidente que los consumidores -jóvenes y adultostienen una gran responsabilidad sobre sus consumos de alcohol y otras drogas y que deben ejercerla. Pero en términos de salud pública sabemos que no todo se puede dejar a la iniciativa y responsabilidad individual, entre otras cosas porque las conductas individuales tienen repercusiones sobre los demás -si alguien por haber bebido tiene un accidente y mata a otra persona se entiende perfectamente que el beber alcohol no puede ser sólo algo que depende de cada individuo. Y en este sentido vemos que el campo de la prevención del alcohol tiene una gran dimensión política, mucho más allá de la que le corresponde al político en el tratamiento, donde la responsabilidad -por lo menos directa- recae mucho más en el profesional y en la persona afectada. Muchas de las medidas preventivas más eficaces hemos podido comprobar que dependen directamente de decisiones políticas.

El profesional tiene la responsabilidad de reunir información, de investigar y de hacer propuestas eficaces y asumibles a los responsables de las políticas. Queda mucho por hacer pero también es ya mucho lo hecho. Una de las prioridades de los profesionales tiene que ser, en la situación actual, tomarse en serio la evaluación de resultados de los programas que se proponen. Esto es especialmente preocupante tanto a nivel de la prevención escolar como de las nuevas estrategias que se están proponiendo de buscar espacios alternativos al consumo de alcohol los fines de semana. No se puede seguir ya avalando como profesionales programas que se extienden por la geografía española que no estén soportados por una evaluación de resultados. Ya no se puede alegar motivos de urgencia. Los problemas están aquí para quedarse. Conocemos cuales son los caminos y 
hay que seguirlos. De no hacerlo así estamos haciendo un flaco favor a la prevención.

\section{BIBLIOGRAFÍA}

(1) ** Plan Nacional sobre Drogas. Informe $n^{\circ} 4$. Observatorio español sobre drogas. Madrid: Ministerio del Interior; 2001.

(2) Wortis J. The perennial alcohol problem. Biological Psychiatry 1993;34:589-90.

(3) NTC Publications. World Drink Trends. Oxfordshire: NTC Publications; 1993.

(4) *** Edwards G; et al. Alcohol y Salud Pública. Barcelona: Prous Science; 1997.

(5) Christianne L, Hupkens H, Ronnal A, Knibbe., Drop MJ. Alcohol Consumption in the European Community: Uniformity and Diversity in Drinking Paterns. Addiction 1993;88:1391-404.

(6) ** Calafat A; Fernández C; Juan M, et al. Risk and control in the recreational drug culture. Palma de Mallorca: Irefrea; 2001.

(7) ** Calafat A; Juan M; Becoña E, et al. Salir de marcha y consumo de drogas. Madrid: Plan Nacional sobre Drogas; 2000.

(8) DiNardo J; Lemieux T. Alcohol, marijuana and American youth: the unintended effects of government regulation. NBER working paper 4212. Cambridge (Massachusetts): National Bureau of Economic Research; 1992.

(9) Chaloupka F, Laixuthai A. Do youths substitute alcohol and marijuana? Some econometric evidence. Eastern Economic Journal 1997;23(3):253-76.

(10) Amengual M, Calafat A, Palmer A. Alcohol, tabaco y drogas en enseñanza media: 19811988-1992. Adicciones 1993;5(2):141-61.

(11) Elzo J, Laespada MT. El alcohol y la noche. Revista de estudios de juventud 1996;37:4554.

(12) Torres MA, Morales E, López L, et al. Estudio de algunos rasgos del alcoholismo juvenil. En Libro de Ponencias. XVIII Jornadas Nacionales. Barcelona: Socidrogalcohol; 1990;

(13) Monrás M. Cambios en la edad de inicio del tratamiento de la dependencia alcohólica. ¿Debemos cambiar la oferta asistencial?". Adicciones 2001;13(2):139-46.
(14) Cahalan D. Galanter M, editors. Studying drinking problems rather than alcoholism. Recent Developments in Alcoholism. New York: Plenum; 1987; . p. 363-72.

(15) Freixa F. Conceptualización del alcoholismo. En Aizpiri J, Marcos JF, editores. Actualización del tratamiento del alcoholismo. Barcelona: Masson; 1996;. p. 1-21.

(16) Campos R, Huertas R. Alcoholismo y degeneración en la medicina positivista española. Rev. Asoc. Esp. Neuropsiquiatría 1992; XII (41): 125-9.

(17) Campos R, Huertas R. El alcoholismo como enfermedad social en la España de la Restauración: problemas de definición. Dynamis.Acta Hispanica ad Medicinae Scientiariumque Historia Illustrandam 1991;11:263-86.

(18) Roig Traver A. Sobre los origines del movimiento antialcohólico en España: El Abstemio. Órgano de la Liga Antialcohólica Española (1910-1920). (no publicado)

(19) Kreitman N. Alcohol consumption and the preventive paradox. Br J Addict. 1986 Jun; 81(3): 353-63.

(19) Oficina Regional Europea de la OMS. Plan Europeo de Acción contra el Alcohol. Rev.Esp.Drogodep. 1996;21(1):9-24.

(20) Norström T. Prevention Strategies and alcohol policy. Addiction 1995;90:515-24.

(21) WORLD HEALTH ORGANIZATION. European Alcohol Action Plan. EUR/ICP/ADA/035. Copenhagen: World Health Organization; 1993.

(22) WORLD HEALTH ORGANIZATION. European Alcohol Action Plan 2000-2005. Copenhagen: World Health Organization; 2000.

(23) Gual A, Colom J. From Paris to Stockholm: where does the European Alcohol Action Plan lead to? Addiction 2001;96:1093-6.

(24) Sloan FA; Stout EM; Whetten-Goldstein K, et al. Drinkers, rivers and bartenders: balancing privates choices and public accountability. Chicago: The University of Chicago Press; 2000.

(25) Effective alcohol control policies. 2-19-1; Stockholm: No publicado; 2001;

(26) Loukomskaia MI. Recent alcohol policies in Russia. Alcologia 1997;9(1):37-42.

(27) Coate D, Grossman M. The effects of alcoholic beverage prices and legal drinking ages on youth alcohol use. J. of Law and Economics 1988;31(1):145-71. 
(28) Grossman M, Coate D, Arluck GM. Holder H, editors. Control Issues in Alcohol Abuse Prevention: Strategies for States and Communities. Greenwich, CT: JAI Press; 1987; Price sensitivity of alcoholic beverages in the United States: Youth alcoholic consumption. p. 169-98.

(29) Kenkel DS. Drinking, driving and deterrence: the social costs of alternative policies. J of Law and Economics 1993;36:877-914.

(30) Saffer H, Chaloupka F. En Chaloupka F, Grossman M, Bickel WK, Saffer H, editores. The economic analysis of substance use and abuse. Chicago: National Bureau of Economics Research; 1999;Demographic differentials in the demand for alcohol and illicit drugs. p. 187-211.

(31) Alvarez C, Montagud C, Ruiz MT. The widening social class gap of preventive health behaviours in Spain. European $\mathrm{J}$ of Public Health $2001 ; 11(2): 225-6$.

(32) Calafat A; Stocco P; Mendes F, et al. Characteristics and Social Representation of Ecstasy in Europe. Palma de Mallorca: IREFREA; 1998.

(33) Moskowitz H; Fiorentino D. A review of the Literature on the Effects of Low Doses of Alcohol on Driving-Related Skills. Washington DC: National Highway Traffic Safety Administration; 2000.

(34) Zador P.L., Krawchuck S.A., Voas RB. Alcoholrelated relative risk of driver fatalities and driver involvement in fatal crashes in relation to driver age and gender: An update using 1996 data. J. of Studies on Alcohol 2000;61:387-95.

(35) NHTSA. Trafic Safety Facts 1999: Alcohol National Center for Statistics and Analysis. Washington DC: NHTSA; 2000.

(36) Hingson R, Heeren T, Winter M. Lower legal blood limits for young drivers. Public Health Reports 1994;109(6):738-44.

(37) Hingson R, Heeren T, Winter M. Lowering state legal blood alcohol limits to $0,08 \%$ : the effect on fatal motor vehicle crashes. American J. of Public Health 1996;86(9):1297-9.

(38) Voas RB, Tippets AS, Fell J. The relationship of alcohol safety laws to drinking drivers in fatal crashes. Accid Anal Prev 2000;32:483-92.

(39) Wagenaar AC. Minimum drinking age and alcohol availability to youth: Issues and research needs. En Hilton ME, Bloss G, editores. Economics and the Prevention of Alcohol-Related Problems. Rockville, MD: National Institute on Alcohol Abuse and Alcoholism; 1993; . p. 175-200.

(40) Allen DN, Sprenkel DG, et al. Reactance theory and alcohol consumption laws: further confirmation among collegiate alcohol consumers. J.of Studies on Alcohol 1994;55:34-40.

(41) Impact of the Ohio administrative license suspension. 42nd Annual Proceedings: Association for the Advancement of Automotive Medicine. Des Palines, IL: AAAM; 1998;

(42) Smart RG. Does alcohol advertising affect overall consumption?. A review of empirical studies. J. of Studies on Alcohol 1988;49:333-40.

(43) Chetwynd J, Coope P, Brodie RJ, Wells E. Impact of cigarette advertising on aggregate demand for cigarettes in New Zealand. Addiction 1988;83:409-14.

(44) Fossey E. Young children and Alcohol: A theory of attitude development. Alcohol and alcoholism 1993;28(4):485-98.

(45) Calafat A. Prevención del alcoholismo: necesidad ineludible y eficaz. Psiquiatría Biológica 1998;5(supl. 1):3-12.

(46) Wilde GJS. Effects of mass media communications on health and safety habits: an overview of issues and evidence. Addiction 1993; 88: 983-96.

(47) Ordoñana JR, Gómez Amor J, Galvañ F. El uso del miedo en los mensajes de salud. Gac Sanit 2000;14(supl 3):45-59.

(48) ** Calafat A; Amengual M. Educación sobre el alcohol. Madrid: Plan Nacional sobre Drogas; 1999.

(49) Calafat A. Hacia una prevención institucionalizada y científica. Adicciones 1998;10(4):291-7.

(50) * * Foxcroft DR, Lister-Sharp D, Lowe G, Breen $R$, and Ireland $D$. Preventive programmes for youth: what works? (Trabajo presentado en Conference on young people and alcohol. 19 al 22 de febrero 2001 en Estocolmo)

(51) Luengo A, Romero E, Gómez JA, Guerra A, Lence M La prevención del consumo de drogas y la conducta antisocial en la escuela: análisis y evaluación de un programa. Madrid. Plan Nacional sobre Droga.1999.

(52) Calafat A, Amengual M, Guimerans C, Rodríguez-Martos A, Ruiz R. "Tu decides": 10 años de un programa de prevención escolar. Adicciones 1995;7(4):509-26.

(53) * Tobler N S. Bukoski WJ, editors.Meta-Analyisis of Drug Abuse Prevention Programs. Rock- 
ville, MD: NIDA; 1997;Meta-Analysis of Adolescence Drug Prevention Programs: Results of the 1993 Meta-Analysis. p. 5-68.

(54) Caulkins JP; Rydell P; Everingham SS, et al. An ounce of prevention: a pound of un uncertainty. Santa Monica (California): RAND; 1999.

(55) Allamani A. Community alcohol action: experiences in Europe. Alcologia 1997;9(1):17-21.

(56) Holder HD; Howard JM; et al. Holder HD and Howard JM, editors.Community prevention trials for alcohol problems. Methodological issues. Westport, CT: Praeger; 1992. 1p.

(57) ** Becoña E. Bases teóricas que sustentan los programas de prevención de drogas. Madrid: Plan Nacional sobre Drogas; 1999.

(58) Holder HD. Summing up: lessons from a comprehensive community prevention trial. Addiction 1997;92(suppl):S293-S301

(59) Spoth R, Lopez Reyes M, Redmond C, Shin C. Assessing a public health approach to delay onset and progression of adolescent substance use: latent transition and log-linear analyses of longitudinal family preventive intervention outcomes. J of Consulting and Clinical Psyhology 1999;67:619-30.

(60) Spoth R, Redmond C, Lepper H. Alcohol initation outcomees of universal family focused preventive interventions: one and two year follow ups of a controled study. J.of Studies on Alcohol 1999;(suppl 13):103-11.
(61) Weitzman ER, Kawachi I. Giving means receiving: the protective effect of social capital on binge drinking on college campuses. American J.of Public Health 2000;90(12):1936-9.

(62) Gentilello LM, Rivara FP, Donovan DM, et al. Alcohol intervention in a trauma center as a means of reducing the risk of injury recurrence. Ann Surgery 1999;230(4):473-83.

(63) Monti PM, Colby SM, Barnett NP, et al. Brief intervention for harm reduction with alcoholpositive older adolescents in a hospital emergency department. J Consult Clin Psychol 1999;67(6):989-94.

(64) Rodríguez-Martos, A, Plasència A, Escayola M, Martí J, Ferrando J, Torralba LI Intervención breve en accidentados con alcoholèmia positiva desde un centro de traumatología Adicciones, 13, 4

(65) C'de Baca J, Lapham SC, Paine S, Skipper BJ. Victim impact panels: Who is sentenced to attend? Does attendance affect recidivism of first time DWI offenders? Alcohol Clin Exp Res 2000;24(9):1420-6.

(66) ** Kilfoyle M; Bellis MA. Club Health. The health of the clubbing Nation. Liverpool: University of Liverpool; 1998.

(67) Calafat A. Evaluation: a key tool for improving drug prevention. Strasbourg: EMCDDA; 2001; 8 , Reviewing the prevention of recreational drug use. p. 87-95. 